\title{
Terapia antibiótica postoperatoria en pacientes sanos sometidos a cirugía de terceros molares impactados.
}

\section{Postoperative antibiotic therapy in healthy patients who underwent impacted third molar surgery.}

\author{
Dusan Marinkovic ${ }^{1,2,3}$, Daphne Azócar ${ }^{1,2,3}$, Luis Romo ${ }^{1,2,3^{*}}$
}

\author{
1. Escuela de Odontología, Facultad de Medicina, \\ Pontificia Universidad Católica de Chile, Santiago, \\ Chile \\ 2. Proyecto Epistemonikos, Santiago, Chile \\ 3. Departamento de Odontología, Facultad de \\ Medicina, Pontificia Universidad Católica de Chile, \\ Santiago, Chile \\ * Correspondencia Autor: Luis Romo | Dirección: \\ Centro Evidencia UC, Pontificia Universidad \\ Católica de Chile, Diagonal Paraguay 476, \\ Santiago, Chile | E-mail: Iromo1966@gmail.com
}

\section{RESUMEN}

Introducción: La adición de tratamiento antibiótico al tratamiento analgésico en el manejo postoperatorio de cirugía de terceros molares en pacientes sanos, ha sido propuesta principalmente para prevenir complicaciones postoperatorias. Sin embargo, es una terapia controvertida en la actualidad. Métodos: Realizamos una búsqueda en Epistemonikos, la mayor base de datos de revisiones sistemáticas en salud, la cual es mantenida mediante el cribado de múltiples fuentes de información, incluyendo MEDLINE, EMBASE, Cochrane, entre otras. Extrajimos los datos desde las revisiones identificadas, analizamos los datos de los estudios primarios, realizamos un metaanálisis y preparamos una tabla de resumen de los resultados utilizando el método GRADE. Resultados y conclusiones: Identificamos siete revisiones sistemáticas que en conjunto incluyeron ocho estudios primarios, de los cuales siete corresponden a ensayos aleatorizados. Concluimos que la adición del tratamiento antibiótico postoperatorio en cirugía de terceros molares en pacientes sanos, disminuye la incidencia de fiebre y probablemente disminuye el desarrollo de infección. Además, podría disminuir la inflamación, pero la certeza de la evidencia es baja. Finalmente, no existe claridad de que la adición de un tratamiento antibiótico postoperatorio en cirugía de terceros molares disminuya el dolor y otros efectos adversos ya que la certeza de la evidencia ha sido evaluada como muy baja.

\begin{abstract}
Introduction: Postoperative antibiotic therapy in addition to analgesics for impacted third molar surgery in healthy patients has been proposed to prevent postoperative complications. However, antibiotic use in healthy patients is still controversial. Methods: We searched in Epistemonikos, the largest database of systematic reviews in health, which is maintained by screening multiple information sources, including MEDLINE, EMBASE, Cochrane, among others. We extracted data from the systematic reviews, reanalyzed data of primary studies, conducted a meta-analysis and generated a summary of findings table using the GRADE approach. Results and conclusions: We identified seven systematic reviews including eight studies overall, of which seven were randomized trials. We conclude that postoperative antibiotic therapy compared with no antibiotic treatment in healthy patients who underwent third molar surgery reduces the risk of fever and probably reduces the risk of infection. Also, it may reduce inflammation, but the certainty of the evidence has been assessed as low. Finally, we are uncertain whether the addition of a postoperative antibiotic in third molar surgery reduces pain and other adverse events, as the certainty of the evidence has been assessed as very low.
\end{abstract}

\section{KEY WORDS}

Third molar surgery; Postoperative antibiotic treatment; Healthy patients Epistemonikos; GRADE.

Int. J. Inter. Dent Vol. 13(3); 186-190, 2020. 


\section{PROBLEMA}

La cirugía de terceros molares es una práctica común en odontología, debido a que la erupción de estos molares puede provocar problemas inflamatorios locales, infecciones y episodios de dolor. Entre los objetivos del tratamiento antibiótico, está lograr la menor cantidad de complicaciones asociadas a la cirugía, tales como dolor, infección, inflamación, entre otros ${ }^{[1]}$.

Dentro de las precauciones y cuidados postoperatorios en cirugía de terceros molares se encuentra la adición de tratamiento antibiótico al tratamiento analgésico para prevenir la aparición de estas complicaciones. Sin embargo es cuestionado, ya que la prescripción excesiva de antibióticos se asocia al desarrollo de resistencia bacteriana, además de diversos efectos adversos asociados al medicamento ${ }^{[2]}$.

Este resumen tiene como objetivo evaluar la efectividad y seguridad del tratamiento antibiótico postoperatorio en pacientes sanos sometidos a cirugía de terceros molares.

\section{MÉTODOS}

Realizamos una búsqueda en Epistemonikos, la mayor base de datos de revisiones sistemáticas en salud, la cual es mantenida mediante búsquedas en múltiples fuentes de información, incluyendo MEDLINE, EMBASE, Cochrane, entre otras. Extrajimos los datos desde las revisiones identificadas y analizamos los datos de los estudios primarios. Con esta información, generamos un resumen estructurado denominado FRISBEE (Friendly Summaries of Body of Evidence using Epistemonikos), siguiendo un formato preestablecido, que incluye mensajes clave, un resumen del conjunto de evidencia (presentado como matriz de evidencia en Epistemonikos), metanálisis del total de los estudios cuando sea posible, una tabla de resumen de resultados con el método GRADE y una sección de otras consideraciones para la toma de decisión.

\section{Mensajes clave}

- El uso de antibióticos reduce la fiebre y probablemente disminuye la infección postoperatoria en cirugía de terceros molares en pacientes sanos.

- El uso de antibióticos podría disminuir la inflamación postoperatoria en cirugía de terceros molares (certeza de la evidencia baja).

- No es posible establecer con claridad si el uso de antibióticos aumenta el riesgo de efectos adversos o si disminuye el dolor postoperatorio en cirugía de terceros molares en pacientes sanos, debido a que la certeza de la evidencia ha sido evaluada como muy baja

Acerca del conjunto de evidencia para esta pregunta

Encontramos siete revisiones sistemáticas ${ }^{[1],[2],[3],[4],[5],[6],[7]}$ que incluyeron ocho estudios primarios ${ }^{[8]}$ [9], [10], [11], [12], [13], [14], [15], de los cuales siete corresponden a ensayos aleatorizados ${ }^{[8], ~[9], ~[10], ~[11], ~[12], ~[13], ~[14] ~}$

Un ensayo fue excluido ${ }^{[9]}$ debido a que la cirugía de terceros molares se realizó específicamente bajo anestesia general y se consideró que los factores inherentes a esta técnica pueden alterar el efecto en los desenlaces estudiados.

La información proveniente del estudio observacional[15] no aumentaba la certeza de la evidencia ni agregaba información adicional relevante, por lo que esta tabla, y el resumen en general están basados en los seis ensayos restantes[[], [10], [11], [12], [13], [14].
Qué tipo de pacientes incluyeron los estudios*

Qué tipo de intervenciones incluyeron los estudios*
Todos los ensayos incluyeron cirugías de terceros molares con algún grado de impactación. Cuatro ensayos ${ }^{[8],[10]}$ [11], [13] incluyeron cirugía de terceros molares inferiores impactados y un ensayo ${ }^{[12]}$ realizó cirugía de terceros molares superiores e inferiores. Cuatro ensayos realizaron cirugía de terceros molares bajo anestesia local ${ }^{[8], ~[10], ~[11], ~[12] ~}$ y en dos ensayos no se especificó el tipo de anestesia fue utilizadaa ${ }^{[13], ~[14] . ~}$

Todos los ensayos incluyeron pacientes sanos, mayores de 18 años y menores de 60 años, con edad promedio entre 20 y 30 años. Cinco ensayos excluyeron a pacientes embarazadas, en periodo de lactancia y a pacientes con alergias a medicamentos ${ }^{[8],[10], ~[11], ~[12], ~[13] ~}$

Todos los ensayos evaluaron la adición de tratamiento antibiótico al tratamiento analgésico postoperatorio en cirugías de terceros molares y compararon con placebo (solo tratamiento analgésico).

Un ensayo ${ }^{[8]}$, utilizó como tratamiento antibiótico metronidazol $400 \mathrm{mg}$ cada ocho horas por cinco días.

Un ensayo ${ }^{[10]}$ usó amoxicilina/ácido clavulánico $500 / 125 \mathrm{mg}$ por vía oral tres veces al día durante cuatro días después de la intervención. Un ensayo ${ }^{[11]}$ utilizó amoxicilina 500 $\mathrm{mg}$ tres veces al día por cinco días y otro ensayo ${ }^{[12]}$ usó amoxicilina 500 mg cada ocho horas por siete días y clindamicina $300 \mathrm{mg}$ cada seis horas por siete días.

Un ensayo ${ }^{[13]}$, utilizó amoxicilina/ácido clavulánico 2000/125 mg por cinco días post operatorios.

Un ensayo[ ${ }^{[14]}$, utilizó amoxicilina 750 mg cada ocho horas por cinco días.

Dos ensayos ${ }^{[8],[11]}$ prescribieron analgesia de ibuprofeno en dosis de $400 \mathrm{mg}$ y $600 \mathrm{mg}$ respectivamente cada 8 horas.

Dos ensayos ${ }^{[10], ~[13] ~ p r e s c r i b i e r o n ~}$ analgesia de metamizol $575 \mathrm{mg}$ cada 8 horas por siete y dos días respectivamente.

Un ensayo ${ }^{[12]}$ no especificó el tipo de analgesia utilizada.

Adicionalmente, un ensayo ${ }^{[10]}$

reporta la indicación de enjuague de clorhexidina $0,12 \% 15 \mathrm{ml}$ cada 8 horas por 7 días post operatorio.

Los ensayos reportaron varios desenlaces, los cuales fueron agrupados por las revisiones sistemáticas de la siguiente manera:

- Infección

- Inflamación

- Fiebre

- Dolor postoperatorio

- Efectos adversos (reacción adversa a medicamentos o resistencia antibiótica)

Cinco ensayos hicieron seguimiento a los siete días [8], [10], [11], [12], [13]. Esta información no se especificó para un ensayo [14]

* La información sobre los estudios primarios es extraída desde las revisiones sistemáticas identificadas, no directamente desde los estudios, a menos que se especifique lo contrario. 


\section{RESUMEN DE LOS RESULTADOS}

La información sobre los efectos del tratamiento antibiótico en cirugía de terceros molares en pacientes sanos, está basada en cinco ensayos aleatorizados que incluyeron 469 pacientes[ ${ }^{[8], ~[10], ~[11], ~[12], ~[13] . ~}$

Todos los ensayos midieron el desenlace infección (469 pacientes), tres ensayos midieron el desenlace efectos adversos (375 pacientes) $)^{[10], ~[11], ~[13] ~}$ dos ensayos midieron el desenlace dolor (306 pacientes) ${ }^{[8],[10]}$ y un ensayo midió el desenlace inflamación (47 pacientes) ${ }^{[8]}$.

El resumen de los resultados es el siguiente:

- El uso de antibiótico probablemente reduce el riesgo de infección postoperatoria en cirugía de terceros molares en pacientes sanos (certeza de la evidencia moderada).
- No es posible establecer con claridad si la adición de tratamiento antibiótico reduce el dolor postoperatorio de cirugía de terceros molares en pacientes sanos, debido a que la certeza de la evidencia existente ha sido evaluada como muy baja.

- El uso de antibióticos podría reducir la inflamación postoperatoria de cirugía de terceros molares en pacientes sanos (certeza de la evidencia baja).

- El uso de antibióticos reduce la fiebre postoperatoria de cirugía de terceros molares en pacientes sanos (certeza de la evidencia alta).

- No es posible establecer con claridad si la adición de tratamiento antibiótico aumenta los efectos adversos postoperatorios en cirugía de terceros molares en pacientes sanos, debido a que la certeza de la evidencia existente ha sido evaluada como muy baja.

\section{Adición de tratamiento antibiótico a tratamiento analgésico postoperatorio de cirugia terceros molares impactados en pacientes sanos}

\begin{tabular}{|c|c|c|c|c|}
\hline $\begin{array}{l}\text { Pacientes } \\
\text { Intervención } \\
\text { Comparación }\end{array}$ & \multicolumn{4}{|c|}{$\begin{array}{l}\text { Pacientes sanos sometidos a cirugía de terceros molares impactados } \\
\text { Adición de tratamiento antibiótico al tratamiento analgésico post } \\
\text { operatorio } \\
\text { No adición (solo tratamiento analgésico) }\end{array}$} \\
\hline \multirow{3}{*}{ Desenlaces } & Efe & luto* & \multirow{3}{*}{$\begin{array}{l}\text { Efecto } \\
\text { relativo } \\
\text { (IC 95\%) }\end{array}$} & \multirow{3}{*}{$\begin{array}{l}\text { Certeza de la } \\
\text { evidencia } \\
\text { (GRADE) }\end{array}$} \\
\hline & $\begin{array}{c}\text { SIN } \\
\text { antibiótico } \\
\end{array}$ & $\begin{array}{c}\text { CON } \\
\text { antibiótico } \\
\end{array}$ & & \\
\hline & \multicolumn{2}{|c|}{ Diferencia: pacientes por 1000} & & \\
\hline \multirow[b]{2}{*}{ Infección } & 116 por 1000 & 17 por 1000 & \multirow{2}{*}{$\begin{array}{l}\text { RR } 0,15 \\
(0,07 \mathrm{a} \\
0,32\end{array}$} & \multirow{2}{*}{$\begin{array}{c}\oplus \oplus \oplus \bigcirc^{1} \\
\text { Moderada }\end{array}$} \\
\hline & \multicolumn{2}{|c|}{$\begin{array}{c}\text { Diferencia: } 99 \text { menos } \\
\text { (Margen de error: } 79 \text { a } 108 \text { menos) }\end{array}$} & & \\
\hline \multirow[b]{2}{*}{ Dolor** } & 106 por 1000 & 55 por 1000 & \multirow{2}{*}{$\begin{array}{l}\text { RR } 0,52 \\
(0,16 \text { a } \\
1,72)\end{array}$} & \multirow{2}{*}{$\begin{array}{l}\oplus \bigcirc \bigcirc \bigcirc^{1,2,3} \\
\text { Muy baja }\end{array}$} \\
\hline & \multicolumn{2}{|c|}{$\begin{array}{c}\text { Diferencia: } 51 \text { menos } \\
\text { (Margen de error: } 89 \text { menos a } 76 \text { más) }\end{array}$} & & \\
\hline \multirow[b]{2}{*}{ Inflamación } & 471 por 1000 & 235 por 1000 & \multirow{2}{*}{$\begin{array}{l}\operatorname{RR} 0,50 \\
(0,24 \text { a } \\
1,02)\end{array}$} & \multirow{2}{*}{$\begin{array}{l}\oplus \oplus \bigcirc \bigcirc^{1,2} \\
\text { Baja }\end{array}$} \\
\hline & \multicolumn{2}{|c|}{$\begin{array}{c}\text { Diferencia: } 236 \text { menos } \\
\text { (Margen de error: } 358 \text { menos a } 9 \text { más) }\end{array}$} & & \\
\hline \multirow[b]{2}{*}{ Fiebre** } & 27 por 1000 & 3 por 1000 & \multirow{2}{*}{$\begin{array}{l}\text { RR } 0,11 \\
(0,01 \text { a } \\
0,88)\end{array}$} & \multirow{2}{*}{$\begin{array}{l}\oplus \oplus \oplus \oplus \\
\text { Alta }\end{array}$} \\
\hline & \multicolumn{2}{|c|}{$\begin{array}{c}\text { Diferencia: } 24 \text { menos } \\
\text { (Margen de error: } 27 \text { menos a } 3 \text { menos) }\end{array}$} & & \\
\hline \multirow{2}{*}{$\begin{array}{l}\text { Efectos } \\
\text { adversos*** }\end{array}$} & 55 por 1000 & 110 por 1000 & \multirow{2}{*}{$\begin{array}{l}\text { RR } 2,00 \\
(0,48 \text { a } \\
8,30)\end{array}$} & \multirow{2}{*}{$\begin{array}{l}\oplus \bigcirc \bigcirc \bigcirc^{3,4} \\
\text { Muy baja }\end{array}$} \\
\hline & \multicolumn{2}{|c|}{$\begin{array}{l}\text { Diferencia: } 55 \text { más } \\
\text { (Margen de error: } 29 \text { menos a } 401 \text { más) }\end{array}$} & & \\
\hline
\end{tabular}

Margen de error: Intervalo de confianza del 95\% (IC 95\%).

RR: Riesgo relativo.

GRADE: Grados de evidencia del GRADE Working Group (ver más adelante).

*Los riesgos SIN antibiótico están basados en los riesgos del grupo control en los estudios. El riesgo CON antibiótico (y su margen de error) está calculado a partir del efecto relativo (y su margen de error).

** Los desenlaces dolor y fiebre fueron evaluados de manera dicotómica al día 6 o 7 de postoperatorio. *** Los efectos adversos reportados fueron resistencia antibiótica y reacciones adversas al medicamento. 
1 Se disminuyó un nivel de certeza de la evidencia por riesgo de sesgo, ya que se identificaron limitaciones relacionadas a la generación de la secuencia de aleatorización, desgaste y reporte selectivo. En el caso del desenlace efectos adversos, se decidió no disminuir por este motivo, dado que la ausencia de sesgo reforzaría la conclusión.

2 Se disminuyó un nivel de certeza de la evidencia por imprecisión, ya que a cada extremo del intervalo de confianza existiría variabilidad en la toma de decisión..

3 Se disminuyó un nivel de certeza de la evidencia por inconsistencia, dado que los distintos ensayos llegaron a conclusiones diferentes (I2 >74\%).

4 Se disminuyó dos niveles de certeza de la evidencia por imprecisión, dado que a cada extremo del intervalo de confianza se tomarían decisiones opuestas.

Siga el enlace para acceder a la versión interactiva de esta tabla (Interactive Summary of Findings - iSoF)

\begin{tabular}{|l|}
\hline Acerca de la certeza de la evidencia (GRADE)* \\
\hline$\oplus \oplus \oplus \oplus$ \\
Alta: La investigación entrega una muy buena indicación del efecto probable. La probabilidad de \\
que el efecto sea sustancialmente distinto† es baja. \\
$\oplus \oplus \oplus \bigcirc$ \\
Moderada: La investigación entrega una buena indicación del efecto probable. La probabilidad de \\
que el efecto sea sustancialmente distinto† es moderada. \\
$\oplus \oplus \bigcirc \bigcirc$ \\
Baja: La investigación entrega alguna indicación del efecto probable. Sin embargo, la probabilidad \\
de que el efecto sea sustancialmente distinto† es alta. \\
$\oplus \bigcirc \bigcirc \bigcirc$ \\
Muy baja: La investigación no entrega una estimación confiable del efecto probable. La \\
probabilidad de que el efecto sea sustancialmente distinto† es muy alta. \\
\hline *Esto es también denominado 'calidad de la evidencia' o 'confianza en los estimadores del efecto'. \\
$\dagger$ Sustancialmente distinto = una diferencia suficientemente grande como para afectar la decisión \\
\hline
\end{tabular}

\section{OTRAS CONSIDERACIONES PARA LA TOMA DE DECISIÓN}

\section{A quién se aplica y a quién no se aplica esta evidencia}

- Esta evidencia aplica a pacientes mayores de 18 años sanos que requieran cirugía de terceros molares superiores e inferiores.

- Si bien los ensayos no contemplaron a menores de edad, no existirían razones clínicas para extrapolar los resultados a pacientes pertenecientes a este grupo que requieran la cirugía.

- La evidencia presentada en este resumen no debería extrapolarse a pacientes embarazadas ni en periodo de lactancia, debido a los distintos requerimientos y riesgos de esta población frente al uso de antibióticos.

\section{Sobre los desenlaces incluidos en este resumen}

- Los desenlaces incluidos en la tabla de resumen de resultados son, según los autores de este resumen, aquellos críticos para la toma de decisión. Esto coincide en general con lo reportado por las revisiones sistemáticas.

\section{Balance daño/beneficio y certeza de la evidencia}

- La evidencia muestra que el uso de antibióticos como tratamiento postoperatoria sería beneficioso para la reducción del riesgo de fiebre e infección luego de una cirugía de terceros molares, y que podría además ayudar en el manejo de la inflamación postoperatoria.

- Los riesgos asociados al tratamiento incluyen el aumento de resistencia antibiótica y desarrollo de efectos adversos, sin embargo la incertidumbre asociada a su efecto no permite determinar el daño real asociado al uso de antibióticos.

- Considerando lo anterior, los autores de este resumen concluyen que no es posible llevar a cabo un adecuado balance entre riesgos y beneficios y que es necesario analizar individualmente las características del paciente e intervención para tomar la decisión clínica.

\section{Consideraciones de recursos}

- Ninguna de las revisiones sistemáticas identificadas realizó un análisis de costo-beneficio.

- La adición de tratamiento antibiótico no tiene un costo adicional importante como para inclinarse en contra de su uso frente a los beneficios observados. Sin embargo, considerando que no existe claridad sobre su seguridad, es necesario contar con un análisis formal de costo efectividad para concluir sobre su uso.

\section{Qué piensan los pacientes y sus tratantes}

- Enfrentados a la evidencia existente, la mayoría de los pacientes y los tratantes podrían inclinarse a favor de la adición de tratamiento antibiótico postoperatorio de cirugía de terceros molares para prevenir fiebre e infección.

- No obstante, algunos pacientes y tratantes podrían inclinarse en contra del uso de antibióticos postoperatorio en pacientes sanos, dado que existe incertidumbre importante en la evidencia sobre su seguridad.

\section{Diferencias entre este resumen y otras fuentes}

- Dos revisiones sistemáticas [2], [4] coinciden con los resultados de este resumen ya que concluyen que la adición de tratamiento antibiótico postoperatorio en cirugía de terceros molares reduce el riesgo de infección. Sin embargo, afirman que faltan estudios sobre la posibilidad del desarrollo de resistencia bacteriana.

- Dos revisiones [5], [6] difieren de las conclusiones de este resumen, ya que concluyen que la adición del tratamiento antibiótico postoperatorio en cirugía de terceros molares no reduce el riesgo de infección en pacientes sanos, por lo tanto su uso debe limitarse a pacientes con factores de riesgo. Sin embargo, estas conclusiones se limitan a un pequeño número de estudios para el análisis estadístico, lo que podría explicar las diferencias en la interpretación de resultados.

- Dos revisiones ${ }^{[1]}{ }^{[3]}$ concluyen que el tamaño del beneficio no es suficiente para recomendar el uso rutinario de tratamiento antibiótico postoperatorio en cirugía de terceros molares, debido al mayor riesgo de efectos adversos leves (diarrea o alergia a los medicamentos) para los pacientes y por la posibilidad de contribuir al desarrollo de resistencia bacteriana.

- Una revisión ${ }^{[7]}$ concluye que la terapia antibiótica perioperatoria tiene beneficios inciertos en pacientes sanos.

- No se encontraron guías de práctica clínicas internacionales 
asociadas al tratamiento postoperatorio de cirugía de terceros molares.

\section{¿Puede que cambie esta información en el futuro?}

- Es improbable que las conclusiones de este resumen cambien frente a nueva evidencia en los desenlaces fiebre e infección. Sin embargo, la probabilidad de que las conclusiones de este resumen cambien es alta para los desenlaces inflamación, dolor y efectos adversos, debido a la incertidumbre existente.

- No se identificaron ensayos ni revisiones sistemáticas en curso en la International Clinical Trials Registry Platform de la Organización Mundial de la Salud o en PROSPERO respectivamente.

\section{CÓMO REALIZAMOS ESTE RESUMEN}

Mediante métodos automatizados y colaborativos recopilamos toda la evidencia relevante para la pregunta de interés y la presentamos en una matriz de evidencia.

Siga el enlace para acceder a la versión interactiva: Terapia antibiótica postoperatoria comparada con no tratamiento antibiótico en pacientes sanos que fueron sometidos a cirugía de terceros molares impactados.

Siga el enlace para acceder a la versión interactiva: Terapia antibiótica postoperatoria comparada con no tratamiento antibiótico en pacientes sanos que fueron sometidos a cirugía de terceros molares impactados.

\section{NOTAS}

Si con posterioridad a la publicación de este resumen se publican nuevas revisiones sistemáticas sobre este tema, en la parte superior de la matriz se mostrará un aviso de "nueva evidencia".

Este artículo es parte del proyecto síntesis de evidencia de Epistemonikos. Se elabora con una metodología preestablecida, siguiendo rigurosos estándares metodológicos y proceso de revisión por pares interno. Cada uno de estos artículos corresponde a un resumen, denominado FRISBEE (Friendly Summary of Body of Evidence using Epistemonikos), cuyo principal objetivo es sintetizar el conjunto de evidencia de una pregunta específica, en un formato amigable a los profesionales clínicos. Sus principales recursos se basan en la matriz de evidencia de Epistemonikos y análisis de resultados usando metodología GRADE. Mayores detalles de los métodos para elaborar este FRISBEE están descritos aquí (http://dx.doi.org/10.5867/medwave.2014.06.5997)

La Fundación Epistemonikos es una organización que busca acercar la información a quienes toman decisiones en salud, mediante el uso de tecnologías. Su principal desarrollo es la base de datos Epistemonikos (www.epistemonikos.org).

\section{DECLARACIÓN DE CONFLICTOS DE INTERESES}

Los autores declaran no tener conflictos de intereses con la materia de este artículo.

\section{AGRADECIMIENTOS}

Este resumen de evidencia fue elaborado con el apoyo metodológico del Centro Evidencia UC, Facultad de Medicina, Pontificia Universidad Católica de Chile.

\section{Bibliografía}

1. Lodi G, Figini L, Sardella A, Carrassi A, Del Fabbro M, Furness S. Antibiotics to prevent complications following tooth extractions. Cochrane Database Syst Rev. 2012 Nov 14:11:CD003811. doi: 10.1002/14651858.CD003811.pub2. PMID: 23152221. 2. Menon RK, Gopinath D, Li KY, Leung YY, Botelho MG. Does the use of amoxicillin/ amoxicillin-clavulanic acid in third molar surgery reduce the risk of postoperative infection? A systematic review with meta-analysis. Int J Oral Maxillofac Surg. 2019 Feb;48(2):263-273. doi: 10.1016/j.jjom.2018.08.002. Epub 2018 Aug 23. PMID: 30145064 .

3. Arteagoitia MI, Barbier L, Santamaría J, Santamaría G, Ramos E. Efficacy of amoxicillin and amoxicillin/clavulanic acid in the prevention of infection and dry socket after third molar extraction. A systematic review and meta-analysis. Med Oral Patol Oral Cir Bucal. 2016 Jul 1;21(4):e494-504. doi: 10.4317/medoral.21139. PMID: 26946211; PMCID: PMC4920465.

4. Ramos E, Santamaría J, Santamaría G, Barbier L, Arteagoitia I. Do systemic antibiotics prevent dry socket and infection after third molar extraction? A systematic review and meta-analysis. Oral Surg Oral Med Oral Pathol Oral Radiol. 2016 Oct;122(4):403-25. doi: 10.1016/j.oooo.2016.04.016. Epub 2016 May 11. PMID: 27499028.

5. Isiordia-Espinoza MA, Aragon-Martinez OH, Bollogna-Molina RE, Alonso-Castro ÁJ. Infection, Alveolar Osteitis, and Adverse Effects Using Metronidazole in Healthy Patients Undergoing Third Molar Surgery: A Meta-analysis. J Maxillofac Oral Surg. 2018 Jun;17(2):142-149. doi: 10.1007/s12663-017-1031-x. Epub 2017 Jul 1. PMID: 29618877; PMCID: PMC5878170.

6. Isiordia-Espinoza MA, Aragon-Martinez OH, Martínez-Morales JF, Zapata-Morales JR. Risk of wound infection and safety profile of amoxicillin in healthy patients which required third molar surgery: a systematic review and meta- analysis. Br J Oral Maxillofac Surg. 2015 Nov;53(9):796-804. doi: 10.1016/j.bjoms.2015.06.013. Epub 2015 Aug 25. PMID: 26316017.

7. Blatt S, Al-Nawas B. A systematic review of latest evidence for antibiotic prophylaxis and therapy in oral and maxillofacial surgery. Infection. 2019 Aug;47(4):519-555. doi: 10.1007/s15010-019-01303-8. Epub 2019 Apr 3. PMID: 30945142.

8. Sekhar $\mathrm{CH}$, Narayanan V, Baig MF. Role of antimicrobials in third molar surgery: prospective, double blind,randomized, placebo-controlled clinical study. $\mathrm{Br} \mathrm{J}$ Oral Maxillofac Surg. 2001 Apr;39(2):134-7. doi: 10.1054/bjom.2000.0557. PMID: 11286448.
9. Kaziro GS. Metronidazole (Flagyl) and Arnica Montana in the prevention of postsurgical complications, a comparative placebo controlled clinical trial. $\mathrm{Br} \mathrm{J}$ Oral Maxillofac Surg. 1984 Feb;22(1):42-9. doi: 10.1016/0266-4356(84)90007-x. PMID: 6365158.

10. Arteagoitia I, Diez A, Barbier L, Santamaría G, Santamaría J. Efficacy of amoxicillin/ clavulanic acid in preventing infectious and inflammatory complications following impacted mandibular third molar extraction. Oral Surg Oral Med Oral Pathol Oral Radio Endod. 2005 Jul;100(1):e11-8. doi: 10.1016/j.tripleo.2005.03.025. PMID: 15953905.

11. López-Cedrún JL, Pijoan JI, Fernández S, Santamaria J, Hernandez G. Efficacy of amoxicillin treatment in preventing postoperative complications in patients undergoing third molar surgery: a prospective, randomized, double-blind controlled study. J Oral Maxillofac Surg. 2011 Jun;69(6):e5-14. doi: 10.1016/j.joms.2011.01.019. Epub 2011 Apr 5. PMID: 21470751.

12. Adde CA, Soares MS, Romano MM, Carnaval TG, Sampaio RM, Aldarvis FP, Federico LR. Clinical and surgical evaluation of the indication of postoperative antibiotic prescription in third molar surgery. Oral Surg Oral Med Oral Pathol Oral Radiol. 2012 Nov;114(5 Suppl):S26-31. doi: 10.1016/j.tripleo.2011.08.018. Epub 2012 Jan 27. PMID: 23083952.

13. Lacasa JM, Jiménez JA, Ferrás V, Bossom M, Sóla-Morales O, García-Rey C, Aguilar L, Garau J. Prophylaxis versus pre-emptive treatment for infective and inflammatory complications of surgical third molar removal: a randomized, double-blind, placebocontrolled, clinical trial with sustained release amoxicillin/clavulanic acid (1000/62.5 mg). Int J Oral Maxillofac Surg. 2007 Apr;36(4):321-7. doi: 10.1016/j.ijom.2006.11.007. Epub 2007 Jan 16. PMID: 17229548.

14. Martín-Ares M, Barona-Dorado C, Martínez-Rodríguez N, Cortés-Bretón-Brinkmann J, Sanz-Alonso J, Martínez-González JM. Does the postoperative administration of antibiotics reduce the symptoms of lower third molar removal? A randomized double blind clinical study. J Clin Exp Dent. 2017 Aug 1;9(8):e1015-e1022. doi: 10.4317/ jced.54024. PMID: 28936293; PMCID: PMC5601102.

15. Lang MS, Gonzalez ML, Dodson TB. Do Antibiotics Decrease the Risk of Inflammatory Complications After Third Molar Removal in Community Practices? J Oral Maxillofac Surg. 2017 Feb;75(2):249-255. doi: 10.1016/j.joms.2016.09.044. Epub 2016 Oct 6. PMID: 28341449. 